\title{
Long non-coding RNA PVT1 activates hepatic stellate cells through competitively binding microRNA-152
}

\author{
Jianjian Zheng ${ }^{1,2}$, Fujun $\mathbf{Y u}^{3}$, Peihong Dong ${ }^{3}$, Limei Wu ${ }^{1}$, Yuan Zhang ${ }^{1}$, Yanwei Hu ${ }^{1}$ \\ and Lei Zheng ${ }^{1}$ \\ ${ }^{1}$ Department of Laboratory Medicine, Nanfang Hospital, Southern Medical University, Guangzhou, Guangdong, P.R. China \\ ${ }^{2}$ Key Laboratory of Surgery, the First Affiliated Hospital of Wenzhou Medical University, Wenzhou, Zhejiang, P.R. China \\ 3 Department of Infectious Diseases, the First Affiliated Hospital of Wenzhou Medical University, Wenzhou, Zhejiang, P.R. \\ China \\ Correspondence to: Lei Zheng, email: nfyyzhenglei@smu.edu.cn \\ Keywords: plasmacytoma variant translocation 1 (PVT1); microRNA-152; DNA methylation; patched1 (PTCH1); Pathology Section \\ Received: May 27, 2016 \\ Accepted: August 25, 2016 \\ Published: August 30, 2016
}

\section{ABSTRACT}

Epithelial-mesenchymal transition (EMT) process is considered as a key event in the activation of hepatic stellate cells (HSCs). Hedgehog (Hh) pathway is known to be required for EMT process. Long non-coding RNAs (IncRNAs) have been reported to be involved in a wide range of biological processes. Plasmacytoma variant translocation 1 (PVT1), a novel IncRNA, is often up-regulated in various human cancers. However, the role of PVT1 in liver fibrosis remains undefined. In this study, PVT1 was increased in fibrotic liver tissues and activated HSCs. Depletion of PVT1 attenuated collagen deposits in vivo. In vitro, PVT1 down-regulation inhibited HSC activation including the reduction of HSC proliferation, a-SMA and type I collagen. Further studies showed that PVT1 knockdown suppressed HSC activation was through inhibiting EMT process and Hh pathway. Patched1 (PTCH1), a negative regulator factor of Hh pathway, was enhanced by PVT1 knockdown. PTCH1 demethylation caused by miR-152 was responsible for the effects of PVT1 knockdown on PTCH1 expression. Notably, miR152 inhibitor reversed the effects of PVT1 knockdown on HSC activation. Luciferase reporter assays and pull-down assays showed a direct interaction between miR-152 and PVT1. Collectively, we demonstrate that PVT1 epigenetically down-regulates PTCH1 expression via competitively binding miR-152, contributing to EMT process in liver fibrosis.

\section{INTRODUCTION}

Liver fibrosis occurs in almost all patients with chronic liver diseases (CLDs) and represents the final common pathway of virtually all types of CLDs [1]. Liver fibrosis is considered as a reversible wound-healing process and featured with the excess deposition of extracellular matrix (ECM) proteins [2]. With prolonged liver injuries in CLDs, fibrosis may progress to cirrhosis and hepatocellular carcinoma (HCC) [3]. Liver fibrosis is a major cause of morbidity and mortality worldwide, therefore, it has been a major public health concern.

The activation and transdifferentiation of hepatic stellate cells (HSCs) are pivotal events in liver fibrosis [1, 4]. To explore the effective treatments on the suppression of activated HSCs is a potential targeted therapy for liver fibrosis. Epithelial-mesenchymal transition (EMT) is a process whereby epithelial cells gradually lose their epithelial signatures, while acquiring the characteristics of mesenchymal cells. Interestingly, EMT process is involved in the activation of HSCs [5]. Activated EMT process contributes to HSC trans-differentiation and liver fibrosis via activating Hedgehog (Hh) signaling pathway [6]. Conversely, Hh pathway inactivation contributes to the suppression of EMT process in HSCs [7]. Patched1 (PTCH1), a member of $\mathrm{Hh}$ family, is a negative regulator of Hh pathway. Our previous study showed that microRNA-152 (miR-152) inhibits liver fibrosis by attenuating DNA methyltransferase 1(DNMT1)-mediated PTCH1 methylation [8]. 
Long non-coding RNAs (lncRNAs) are a class of transcripts ( $>200 \mathrm{nt}$ in length) that structurally resemble mRNAs but do not encode proteins. Emerging evidence has demonstrated that IncRNAs are frequently deregulated in various diseases and implicated in the development and metastasis of cancers [9]. The roles of lncRNAs are involved in multiple biological processes such as proliferation, apoptosis, cell migration and differentiation [9, 10]. Although many lncRNAs have been reported to be involved in the activation of HSCs $[11,12]$, the specific roles of lncRNAs in regulating EMT in liver fibrosis remain unclear. Recently, plasmacytoma variant translocation 1 (PVT1), a novel lncRNA, has been reported to be up-regulated in human cancers such as HCC, ovarian cancer and non-small lung cancer [1315]. PVT1 may serve as a crucial regulator in cancers. For example, PVT1 can promote cell proliferation, cell cycling, and the acquisition of stem cell-like properties in HCC cells by stabilizing NOP2 protein [13]. Increased PVT1 level may be associated with poor prognosis in many cancers [16, 17]. Notably, PVT1 can serve as a mediator of ECM accumulation in the kidney [18], suggesting that PVT1 may be involved in fibrosis. In this study, we aimed to explore the roles of PVT1 in liver fibrosis.

\section{RESULTS}

\section{Up-regulation of PVT1 in activated HSCs and liver fibrotic tissues}

It is known that isolated mouse HSCs cultured on plastic will gradually become an activated myofibroblastic phenotype during culture days [19]. To explore the biological role of PVT1 in liver fibrosis, we firstly detected the expression of PVT1 in primary 2-dayold HSCs and primary 10-day-old HSCs. As indicated by Figure 1A, there are three different PVT1 isoforms

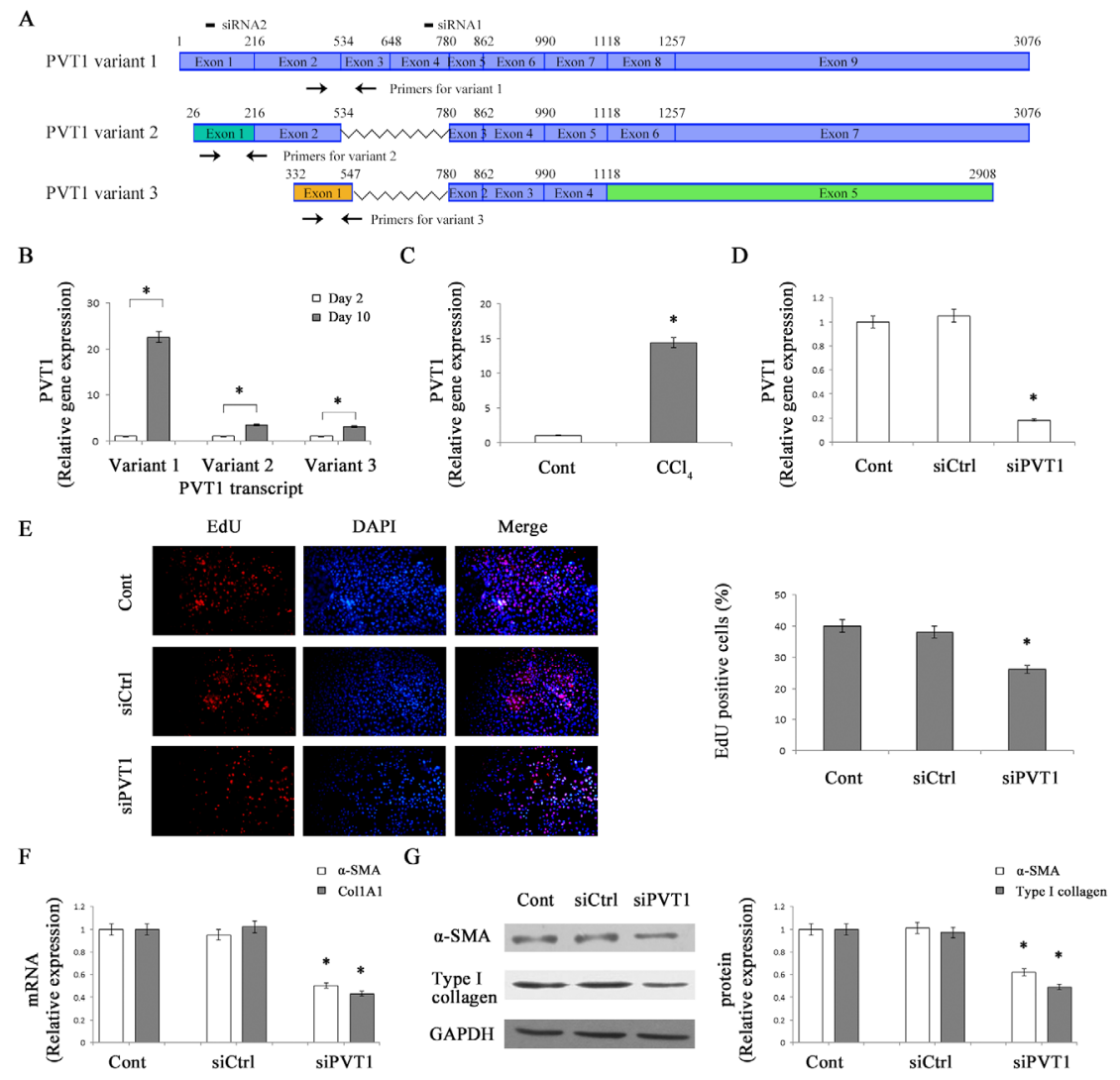

Figure 1: Up-regulation of PVT1 in liver fibrosis and effects of PVT1 on HSC activation in vitro. A. Schematic diagram of variants of the PVT1 transcript. Color boxes indicated the exons of variants of the PVT1 transcript. Black boxes indicated the siRNAs for PVT1 transcript variant 1. Black arrows indicated the PCR primers in the variants. Notably, the sequences of the exon 1 of PVT1 transcript variant 2 were different from those in PVT1 transcript variant 1. Moreover, the sequences of the exon 1 and exon 5 of PVT1 transcript variant 3 were different from those in PVT1 transcript variant 1 and 2. B. PVT1 transcript variant 1, PVT1 transcript variant 2 and PVT1 transcript variant 3 expressions were detected by qRT-PCR in primary HSCs. C. PVT1 was analyzed by qRT-PCR in CCl ${ }_{4}$ mice. D. PVT1 was measured by qRT-PCR in primary HSCs transfected with siPVT1. E. Cell proliferation was determined by the EdU assay. The mRNA F. and protein G. expression levels of $\alpha$-SMA and type I collagen were analyzed in primary HSCs transfected with siPVT1. GAPDH was used as internal control. ${ }^{*} P<0.05$ compared to the control. Each value is the mean $\pm \mathrm{SD}$ of three experiments. 
including PVT1 transcript variant 1 (NR_003368.2), PVT1 transcript variant 2 (NR_132746.1) and PVT1 transcript variant 3 (NR_132747.1) in NCBI (National Center for Biotechnology Information) database. As shown by quantitative real-time PCR (qRT-PCR) analysis, the expressions of all PVT1 variants were up-regulated at day 10 compared with those at day 2 (Figure 1B). Notably, PVT1 variant 1 is mostly up-regulated than PVT1 variant 2 and 3 in activated HSCs. Thus, PVT1 variant 1 was selected for the following analysis. Moreover, PVT1 expression was analyzed in carbon tetrachloride $\left(\mathrm{CCl}_{4}\right)$ induced liver fibrosis in mice. Compared with control liver, PVT1 expression in $\mathrm{CCl}_{4}$-treated liver was increased by 14.4-fold (Figure 1C). Our data suggest that PVT1 was increased during liver fibrosis and may play a role in liver fibrosis.

\section{Knockdown of PVT1 inhibits the activation of HSCs}

HSC activation is characterized by enhanced cell proliferation, over-production of ECM, and de novo synthesis of $\alpha$-smooth muscle actin ( $\alpha$-SMA) [20]. To evaluate the functions of PVT1 in liver fibrosis, primary HSCs were transfected with PVT1 siRNA (siPVT1). It was found that PVT1 level in HSCs transfected with SiPVT1 was decreased by $82 \%$ relative to the control (Figure 1D). Using 5-Ethyny-2'-deoxyuridine (EdU) assays, the effects of siPVT1 on HSC proliferation were examined. PVT1 knockdown led to a significant reduction in cell proliferation (Figure 1E). Next, we examined the roles of siPVT1 on $\alpha$-SMA and collagen expression. The mRNA levels of $\alpha$-SMA and alpha-1(I) collagen (Col1A1) were decreased by $50 \%$ and $57 \%$, respectively, in siPVT1 group relative to the control (Figure 1F). Consistent with mRNA data, immunoblot analysis showed that the protein levels of $\alpha$-SMA and type I collagen in cells transfected with siPVT1 were reduced by $38 \%$ and $51 \%$, respectively (Figure 1G). To further confirm the effects of PVT1 knockdown on $\alpha$-SMA and type I collagen, PVT1 siRNA2 (siPVT1-2) was transfected into HSCs. As shown in Figure S1A, PVT1 expression was reduced in siPVT1-2 group. Consistent with the previous results, siPVT1-2 resulted in a significant reduction in the protein levels of $\alpha$-SMA and typeIcollagen (Figure S1B). Our results suggest that PVT1 contributes to HSC activation.

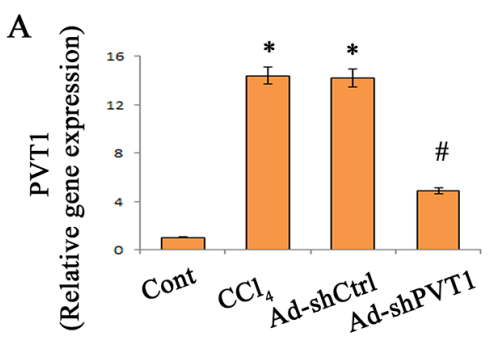

B

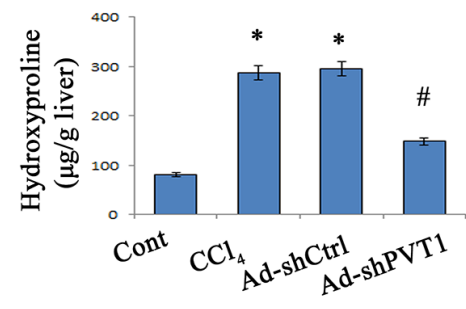

$\mathrm{C}$

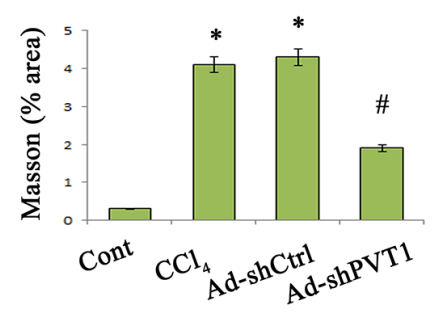

D
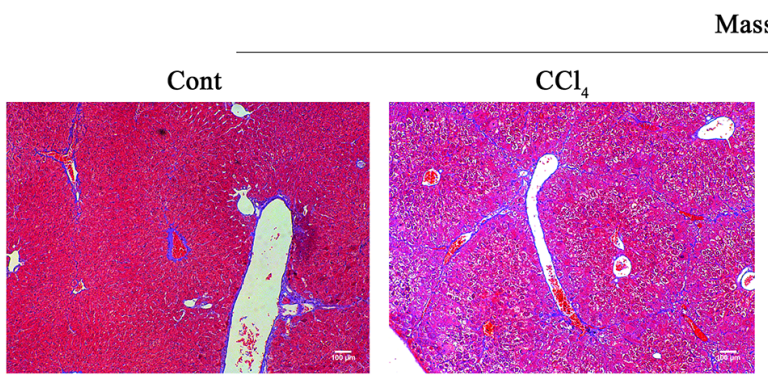

Masson
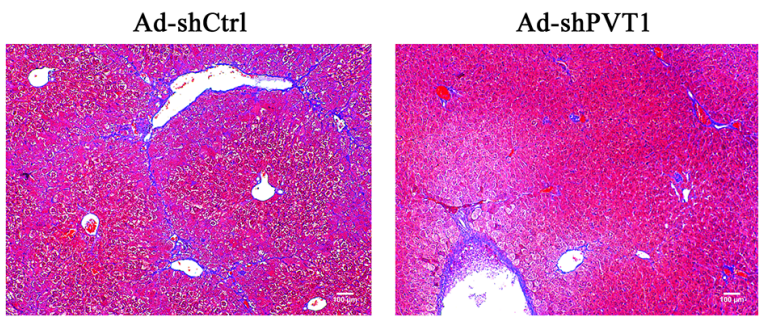

$\mathrm{E}$

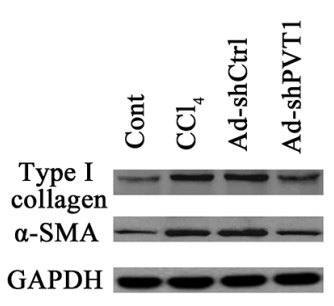

$\mathbf{F}$
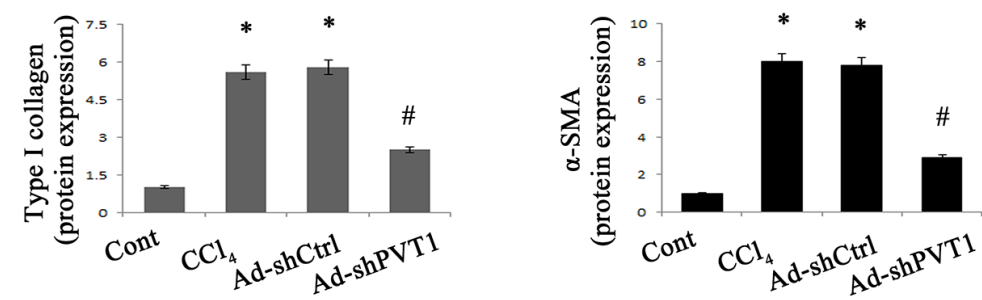

Figure 2: Silencing PVT1 contributes to the suppression of liver fibrosis in $\mathbf{C C l}_{4}$ mice. A. Relative gene expression of PVT1 was analyzed by qRT-PCR. B. The level of hydroxyproline was detected in $\mathrm{CCl}_{4}$ mice after Ad-shPVT1 treatment. (C and D) Accumulation of collagen was assessed by Masson staining. Scale bar, $100 \mu \mathrm{m}$. (E and F) The protein levels of $\alpha$-SMA and type I collagen were measured by Western blotting. GAPDH was used as internal control. ${ }^{*} P<0.05$ compared to the control and ${ }^{\#} P<0.05$ compared to the $\mathrm{CCl}_{4}$ group. Each value is the mean $\pm \mathrm{SD}$ of three experiments. 


\section{Loss of PVT1 alleviates CCl4-induced liver fibrosis}

To confirm the role of PVT1 in the progression of liver fibrosis in vivo, the effects of PVT1 knockdown on $\mathrm{CCl}_{4}$-induced liver fibrosis were explored. Delivery of adenoviral vectors expressing shRNA against PVT1 (Ad-shPVT1) significantly led to the suppression of PVT1 expression in vivo (Figure 2A). As shown by liver hydroxyproline content and Masson staining, $\mathrm{CCl}_{4}^{-}$ induced collagen expression was inhibited by loss of PVT1 (Figure 2B-2D). To further confirm the effects of loss of PVT1 on collagen expression, $\mathrm{CCl}_{4}$ mice were also treated with adenoviral vectors expressing shRNA2 against PVT1 (Ad-shPVT1-2). Notably, Ad-shPVT1-2 led to a significant reduction in hydroxyproline content in
$\mathrm{CCl}_{4}$ mice (Figure S2). In line with these results, increased type I collagen expression caused by $\mathrm{CCl}_{4}$ was blocked down by PVT1 knockdown (Figure 2E and Figure 2F). Likewise, silencing PVT1 resulted in the suppression of $\mathrm{CCl}_{4}$-induced $\alpha$-SMA expression (Figure 2E and Figure $2 \mathrm{~F})$.

\section{Silencing PVT1 attenuates liver fibrosis through suppressing EMT}

Next, to investigate whether EMT process was involved in the effects of PVT1, the levels of EMT markers such as E-cadherin (epithelial marker), desmin and vimentin (myofibroblastic marker) were detected in primary HSCs. The mRNA and protein expressions of E-cadherin were obviously enhanced by loss of PVT1
A

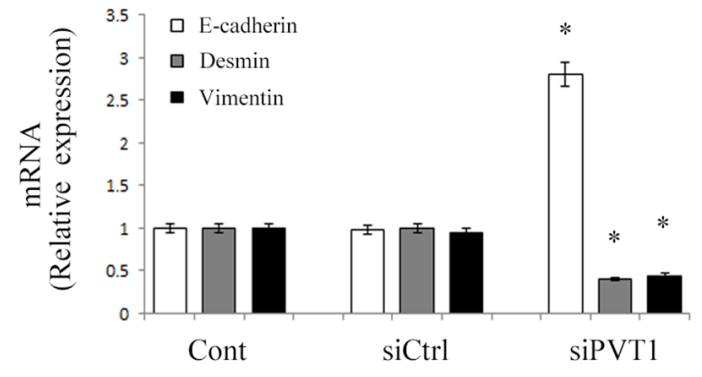

B

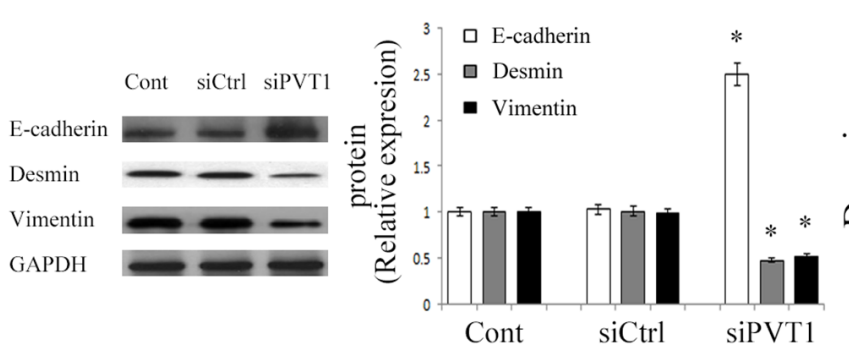

C

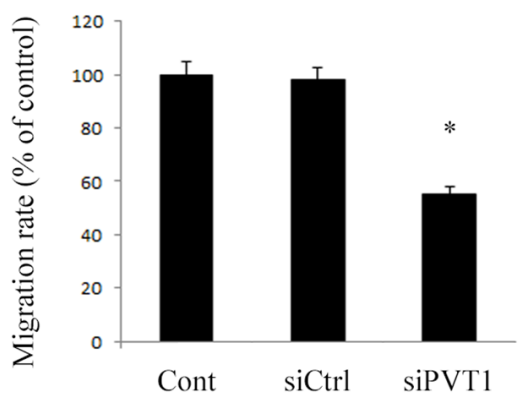

$\mathrm{D}$

Cont
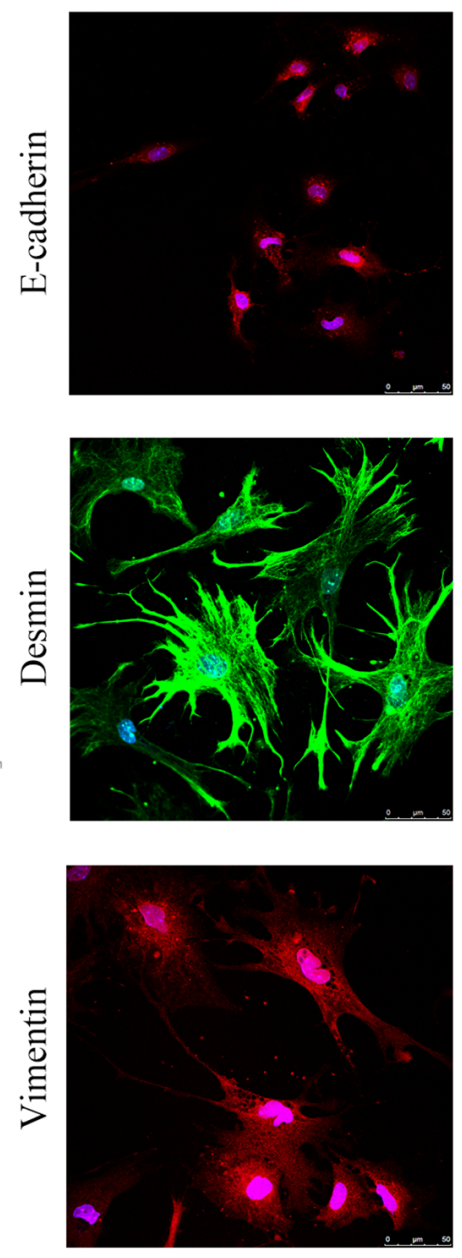

siPVT1
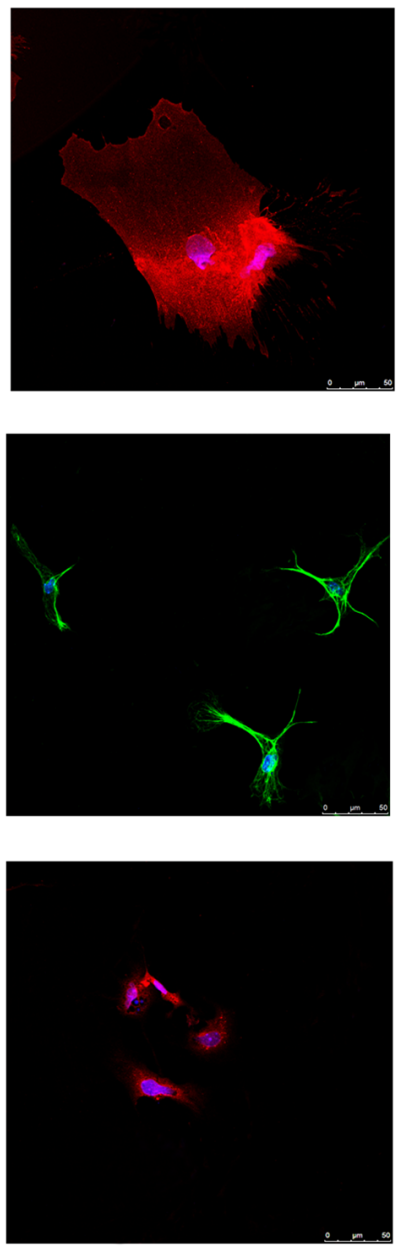

Figure 3: Effects of PVT1 on EMT process. A. The mRNA levels of E-cadherin, desmin and vimentin were analyzed by qRT-PCR. B. The protein levels of E-cadherin, desmin and vimentin were analyzed by Western blotting. GAPDH was used as internal control. C. Cell migration was analyzed by transwell migration assay. Five fields of migrated cells in the lower side of transwell were counted with a microscope at $\times 100$. D. Immunofluorescence staining for E-cadherin (red), desmin (green) and vimentin (red) were evaluated by confocal laser microscopy. DAPI stained nuclei blue. Scale bar, $50 \mu \mathrm{m} .{ }^{*} P<0.05$ compared to the control. Each value is the mean $\pm \mathrm{SD}$ of three experiments. 
(Figure 3A and Figure 3B). Conversely, in comparison with control, the levels of desmin and vimentin were significantly reduced by the inhibition of PVT1 (Figure 3A and Figure 3B). Similarly, E-cadherin expression was enhanced by siPVT1-2, whereas desmin and vimentin expressions were inhibited by siPVT1-2 (Figure S1C). Then, we determined the functions of siPVT1 in cell migration. As confirmed by transwell migration assays, HSCs migration was significantly reduced by PVT1 knockdown (Figure 3C). Using immunofluorescence analysis, we further confirm the effects of siPVT1 on EMT markers. Consistent with the previous results, E-cadherin expression was increased by siPVT1, whereas the levels of desmin and vimentin were reduced in cells after siPVT1 treatment (Figure 3D). These data suggest that PVT1 promotes EMT process in liver fibrosis.

\section{Silencing PVT1 inhibits Hh pathway and PTCH1 methylation}

The Hh family mainly includes Sonic Hh (SHH), PTCH1, the Smoothened (SMO) and GLI family zinc finger (GLI). To determine whether Hh pathway was involved in the effects of PVT1 on regulating EMT in liver fibrosis, Hh pathway-related genes including PTCH1, SMO and GLI2 were detected by qRT-PCR and immunoblot analysis. The expression of PTCH1 was markedly increased by siPVT1, whereas the expressions of SMO and GLI2 were reduced by siPVT1 (Figure 4A and Figure 4B). In line with these results, siPVT1-2 contributed to the increase in $\mathrm{PTCH} 1$ and the reduction in SMO and GLI2 (Figure S1D). All these results indicated
A

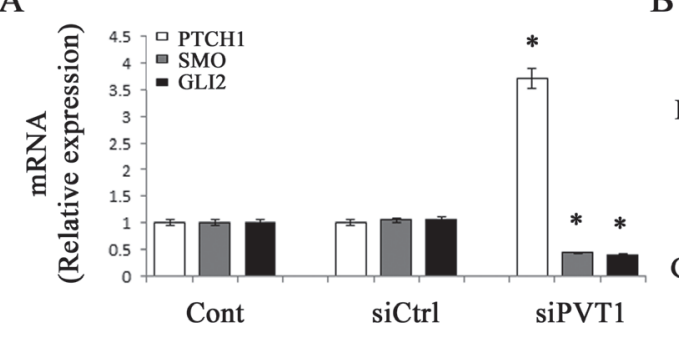

B

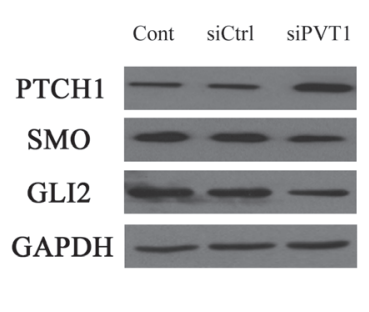

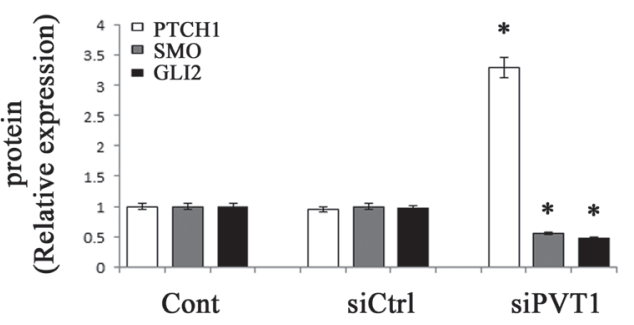

C

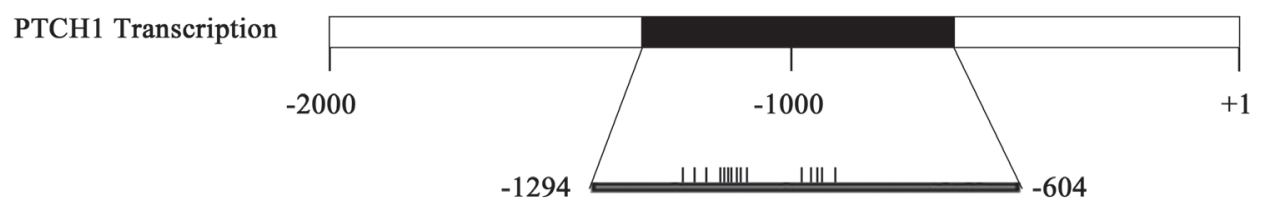

$\mathrm{D}$

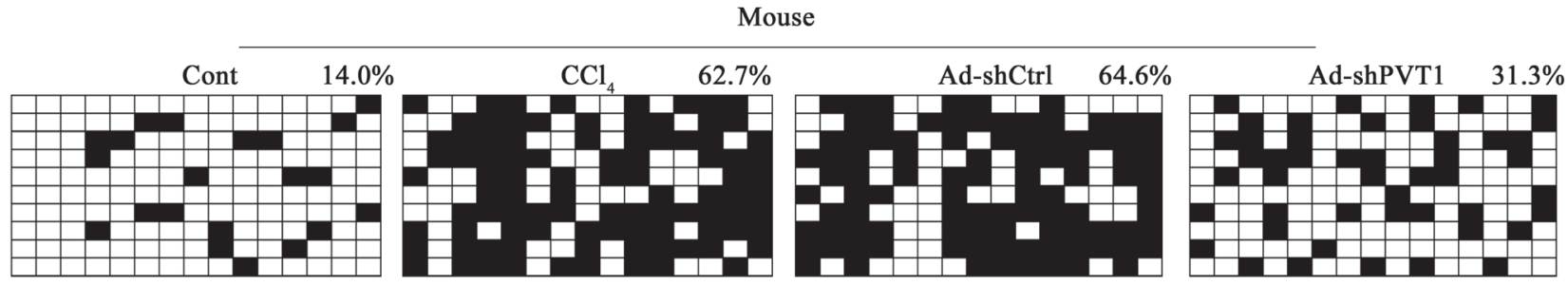

$\mathrm{E}$

Primary HSCs
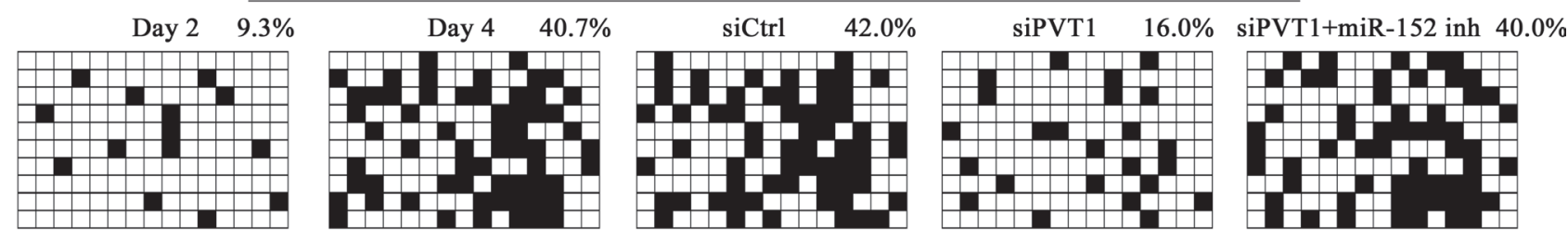

Figure 4: Effects of PVT1 on Hh pathway and PTCH1 promoter methylation. The mRNA A. and protein B. levels of PTCH1, SMO and GLI2 were analyzed by qRT-PCR and Western blotting, respectively. C. A schematic representation of the promoter region amplified by bisulfide sequencing. Black box represents the region selected for bisulfite sequencing. Each vertical bar represents the presence of a $\mathrm{CpG}$ dinucleotide. Promoter methylation of PTCH1 was detected by bisulfide sequencing in mice D. and primary HSCs E. Primary 2-day-old HSCs were transfected with siPVT1 for $48 \mathrm{~h}$ and then treated with miR-152 inhibitor for additional $48 \mathrm{~h}$. The average percentage of DNA methylation was shown at the end of each row. ${ }^{*} P<0.05$ compared to the control. Each value is the mean \pm SD of three experiments. 
that PVT1 knockdown led to Hh pathway inactivation. Recently, Yang et al. found that silencing of PTCH1 level was responsible for the activation of $\mathrm{Hh}$ pathway and associated with PTCH1 methylation in liver fibrosis [21]. To understand whether increased PTCH1 caused by siPVT1 was associated with DNA methylation of PTCH1 promoter, the methylation level at $15 \mathrm{CpG}$ sites within the $\mathrm{CpG}$ island in the PTCH1 locus was analyzed by bisulfite-sequencing analysis (Figure 4C). The average rate of $\mathrm{PTCH} 1$ methylation was $14.0 \%$ in the control mice, whereas it was increased to $62.7 \%$ in $\mathrm{CCl}_{4}$-treated mice (Figure 4D). But Ad-shPVT1 treatment inhibited $\mathrm{CCl}_{4}-$ induced PTCH1 methylation, which was just $31.3 \%$ in AdshPVT1 group (Figure 4D). Similar results were observed in vitro. PTCH1 methylation was increased in primary HSCs during culture days. The average rate of PTCH1 methylation was $9.3 \%$ at Day 2, whereas it was increased to $40.7 \%$ at Day 4 (Figure $4 \mathrm{E}$ ), indicating that PTCH1 methylation was increasing during HSC activation. Notably, PTCH1 methylation rate was reduced to $16 \%$ in siPVT1 group relative to the control (Figure 4E). Taken together, PVT1 promotes EMT process and Hh pathway activation through PTCH1 methylation.

\section{PVT1 enhances PTCH1 methylation via miR-152}

Previously, we demonstrated that DNMT1 is a direct target of miR-152 and miR-152 contributes to the hypomethylation of PTCH1 via inhibiting DNMT1 [8]. Based on these, we next investigate whether miR-152 was involved in siPVT1-induced PTCH1. We firstly detected miR-152 level in primary HSCs during culture days. miR152 level was decreased by $79 \%$ at day 10 relative to day 2 (Figure 5A). Similarly, miR-152 level was reduced in $\mathrm{CCl}_{4}$ group (Figure 5B). miR-152 level may be negatively correlated with PVT1 expression in liver fibrosis. The correlation between miR-152 and PVT1 was further determined in cells transfected with siPVT1. Loss of PVT1 resulted in the elevation of miR-152 level (Figure $5 \mathrm{C})$. Further studies were performed to examine the role of miR-152 in the effect of siPVT1 on HSC activation. Interestingly, miR-152 inhibitor reversed siPVT1suppressed HSC proliferation (Figure 5D). Likewise, reduced $\alpha$-SMA and collagen caused by siPVT1 were inhibited by miR-152 inhibitor (Figure 5E and Figure 5F). Notably, siPVT1-induced PTCH1 was blocked down by

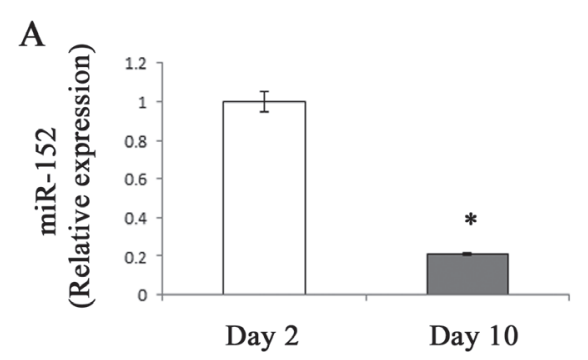

D

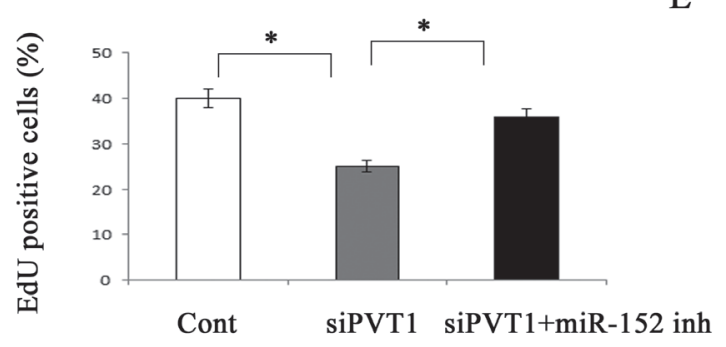

F

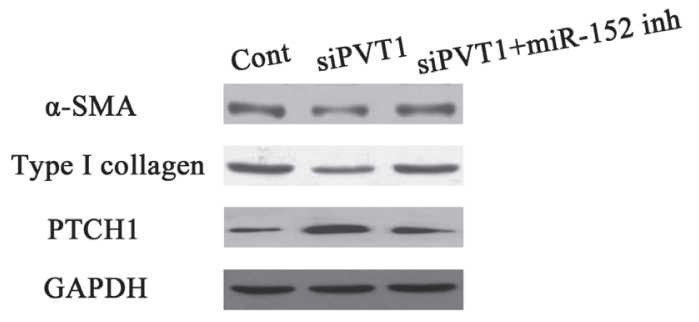

E
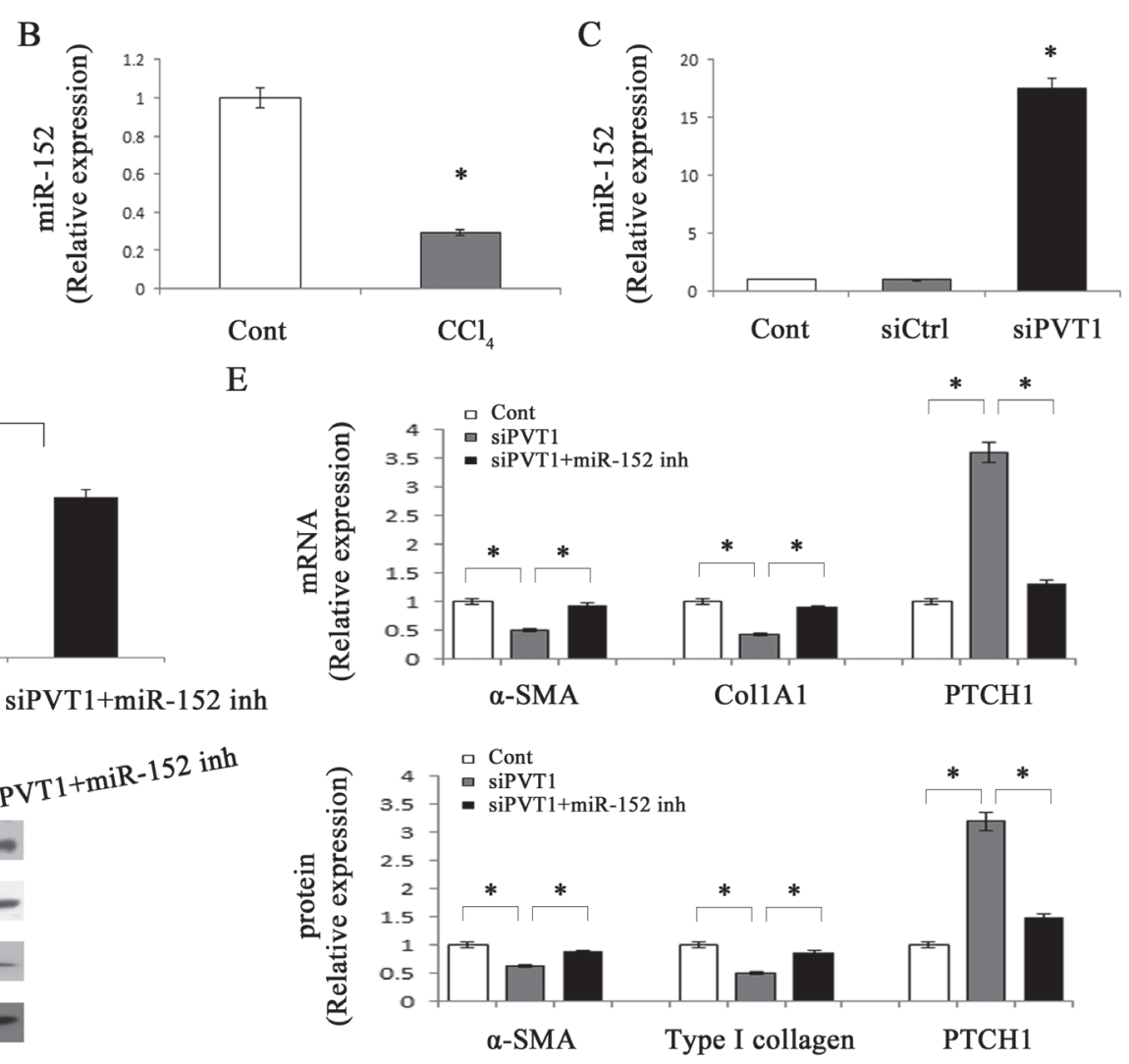

Figure 5: miR-152 is involved in the effects of PVT1 on HSC activation. Primary 2-day-old HSCs were transfected with siPVT1 for $48 \mathrm{~h}$ and then treated with miR-152 inhibitor for additional $48 \mathrm{~h}$. The level of miR-152 was detected by qRT-PCR in primary HSCs A. and $\mathrm{CCl}_{4}$ mice B.. C. The level of miR-152 was measured by qRT-PCR in primary HSCs transfected with siPVT1. D. Cell proliferation was determined by the EdU assay. The mRNA E. and protein F. expression levels of $\alpha$-SMA, type I collagen and PTCH1 were analyzed by qRT-PCR and Western blotting, respectively. GAPDH was used as internal control. $* P<0.05$ compared to the control. Each value is the mean \pm SD of three experiments. 
miR-152 inhibitor (Figure 5E and Figure 5F). Consistent with these, the hypermethylation of PTCH1 was restored by miR-152 inhibitor in siPVT1-transfected cells (Figure 4E). Our data suggest that PVT1 may modulate PTCH1 methylation via miR-152. Bioinformatic analysis (RNA22) shows that PVT1 contains one target site for miR-152 (Figure 6A), indicating that there may be an interaction between miR-152 and PVT1. Then, pmirGLO construct was used to generate a PVT1 luciferase reporter containing the miR-152-binding sites (pmirGLO-PVT1$\mathrm{Wt}$ ) or mutated sites (pmirGLO-PVT1-Mut) (Figure 6A). Our results demonstrated that miR-152 mimics led to the reduction of luciferase activity of pmirGLO-PVT1-Wt in primary HSCs without affecting that of pmirGLOPVT1-Mut (Figure 6B). The results suggest that PVT1 is a target of miR-152. In addition, miR-152 mimics induced a reduction in PVT1 expression, whereas miR-152 inhibitor caused an increase in PVT1 expression (Figure 6C). Interestingly, pri-miR-152 level was not affected by siPVT1 (Figure 6D), suggesting that PVT1 regulates miR152 at the post-transcriptional level. To further determine the direct interaction between miR-152 and PVT1, biotinylated miR-152 (Bio-miR-152) pull-down assay was performed to confirm whether miR-152 could pull down PVT1. As shown in Figure 6E and Figure 6F, Bio-miR152-Wt pulled down PVT1 while Bio-miR-152-Mut had no effect on PVT1, indicating a direct interaction between miR-152 and PVT1. Collectively, PVT1 inhibits PTCH1 expression via competitively binding miR-152.

A

B
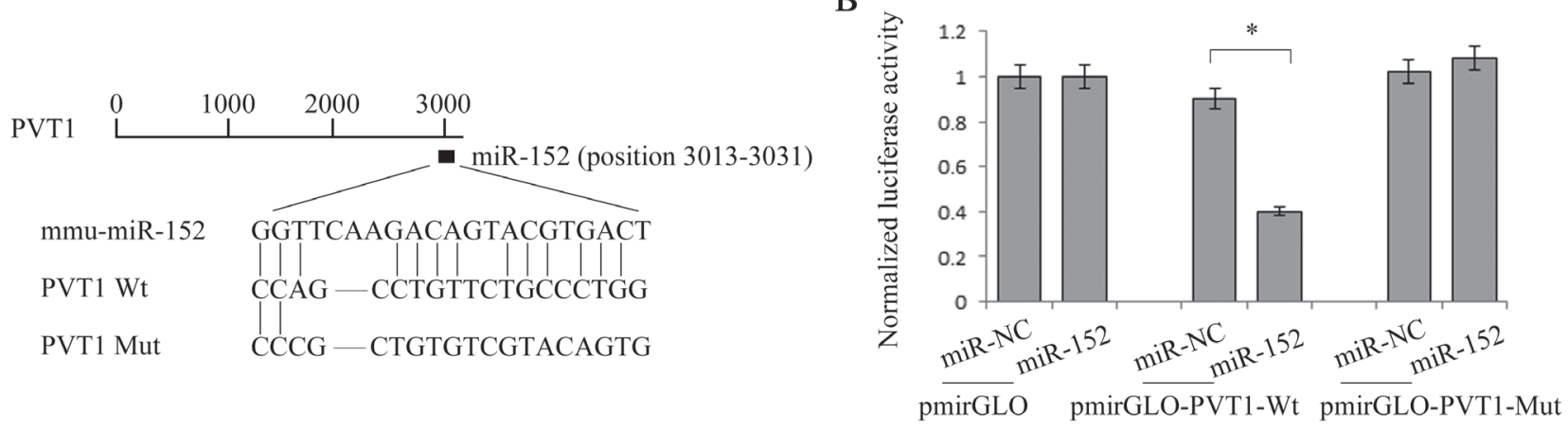

$\mathrm{C}$

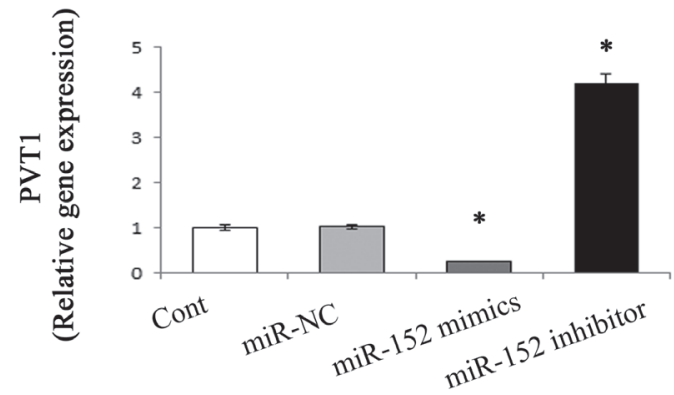

D

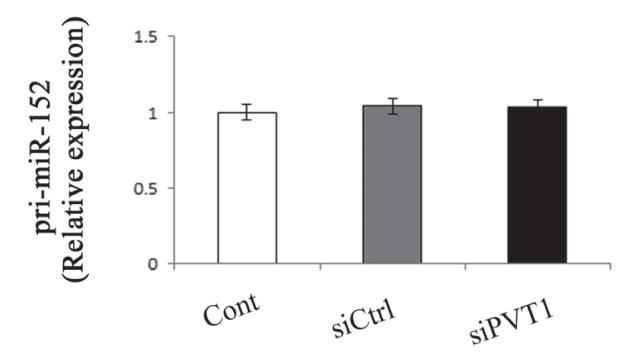

$\mathrm{E}$

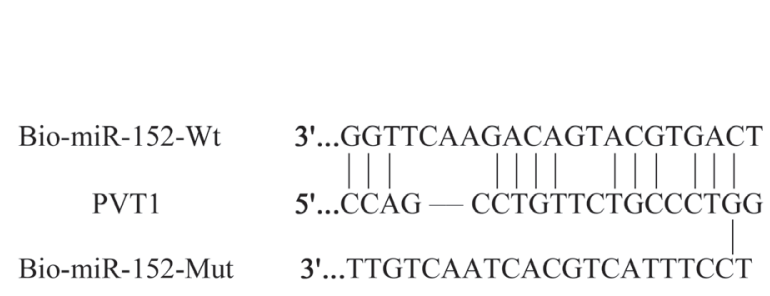

$\mathrm{F}$

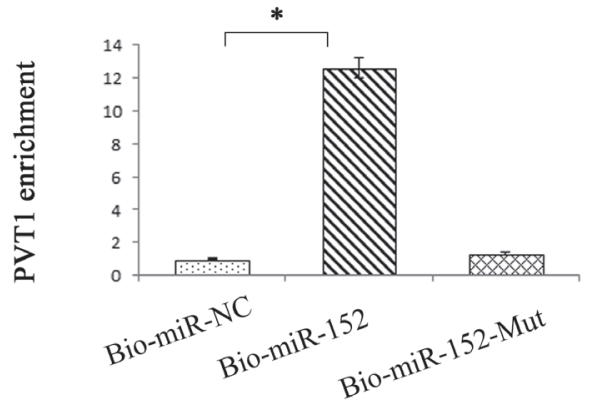

Figure 6: Interaction between PVT1 and miR-152. A. Schematic diagram of the miR-152 binding site in the PVT1 based on RNA22 software. B. Relative luciferase activities of luciferase reporters harboring the wild-type or mutant PVT1 were analyzed $48 \mathrm{~h}$ following transfection with the miR-152 mimics or miR-NC. C. Relative gene expression of PVT1 was analyzed by qRT-PCR in cells transfected with miR-152 mimics or inhibitor. D. Relative gene expression of pri-miR-152 was analyzed by qRT-PCR in cells transfected with siPVT1. E. Schematic diagram of wild type and the mutated form of miR-152 sequence. F. Pull down assay to validate the direct interaction between PVT1 and miR-152. Bio-miR-NC is not complementary to PVT1. ${ }^{*} P<0.05$ compared to the control. Each value is the mean \pm SD of three experiments. 


\section{DISCUSSION}

In this study, we found that PVT1 was increased in fibrotic liver tissues and in activated HSCs. Silencing PVT1 not only inhibited liver fibrosis in vivo but also caused the suppression of activated HSCs in vitro. Due to the loss of PVT1, Hh pathway was inactivated and EMT process was inhibited. Our results suggest that PVT1 plays a pro-fibrotic role in liver fibrosis and this is a first report. We also demonstrate that epigenetic modification of PTCH1 mediated by miR-152 is involved in the effects of PVT1 on HSC activation.

In past decades, IncRNAs have been considered as simply transcriptional "noise" or cloning artifacts. In fact, recent studies have identified large numbers of lncRNAs playing important regulatory roles that were previously reserved for proteins [22]. Recently, emerging evidences have demonstrated that lncRNAs act as important protagonists of cellular functions via diverse molecular mechanisms including chromatin modification, transcriptional regulation and post-transcriptional regulation [10, 11, 22]. Among post-transcriptional regulation, lncRNAs act as competing endogenous
RNAs (ceRNAs) to sponge miRNAs, consequently modulating the de-repression of miRNA targets [23-27]. For example, lncRNA-growth arrest-specific transcript 5 (IncRNA-GAS5) has been shown to increase p27 protein by functioning as a ceRNA for miR-222 in liver fibrosis, resulting in the suppression of liver fibrosis [26]. Recent studies have reported that PVT1 functions as an oncogene and accelerates the progression of cancers $[13,28]$. Wang et al. showed that PVT1 expression is distributed in both nucleus and cytoplasm [13], indicating that PVT1 may serve as a ceRNA for miRNAs. In normal breast, Paci et al. identified PVT1 as a ceRNA for miR-200 family [29]. In this study, whether PVT1 can function as a ceRNA for miRNAs in liver fibrosis was explored. Our results showed that PVT1 enhanced PTCH1 methylation and caused a reduction in PTCH1 expression via competitively binding miR-152. As confirmed by luciferase reporter assays and pull-down assays, there is a direct interaction between miR-152 and PVT1. In addition, PVT1 inhibits miR-152 in a post-transcriptional manner. Notably, all the effects of siPVT1 on HSC activation can be blocked down by miR152 inhibitor. All the data above suggest that PVT1 can function as a ceRNA for miR-152.

DNA methylation is a type of epigenetic

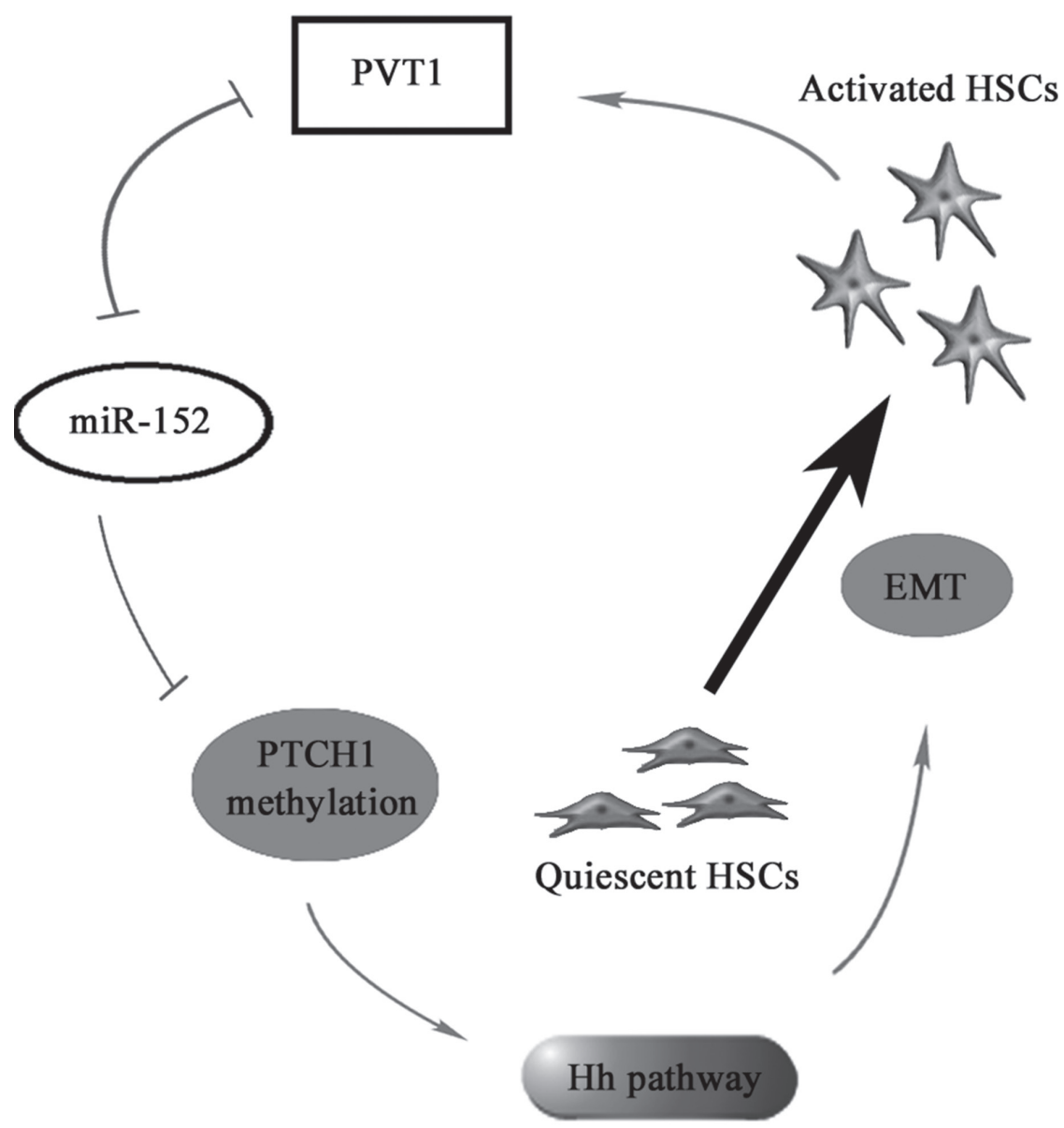

Figure 7: The signaling pathway was discovered in activated HSCs. PVT1 induces miR-152 down-regulation, PTCH1 methylation and activation of Hh pathway, then promotes EMT process, which contributes to the activation of HSCs. 
modifications in mammals and its correlation to pathogenesis of liver fibrosis has been well established [30, 31]. Mammalian DNA is dominantly methylated in the C-5-position of complimentary CpG bp DNMTs, including DNMT1, DNMT3a and DNMT3b [32]. DNA methylation contributes to the loss of PTCH1 in tumors [33]. Yang et al. reported that decreased PTCH1 expression in liver fibrosis is associated with PTCH1 hypermethylation [21]. In our study, PTCH1 hypermethylation in fibrotic liver tissues and activated HSCs was inhibited by PVT1 knockdown. Owing to the loss of PTCH1 methylation caused by PVT1 knockdown, the level of PTCH1 was restored, leading to the inactivation of Hh pathway and the inhibition of EMT process. Interestingly, siPVT1-induced PTCH1 demethylation was inhibited by miR-152 inhibitor. Our results indicated that miR-152 was responsible for the effects of PVT1 on PTCH1 methylation, which was consistent with our previous study [8]. With the restoration of PTCH1 methylation, miR-152 inhibitor reversed siPVT1-inhibited HSC activation. Taken together, PTCH1 expression is associated with its promoter methylation level and PVT1 contributes to HSC activation through regulation of miR-152 and PTCH1 methylation.

In conclusion, we demonstrate that PVT1 can epigenetically inhibit PTCH1 via competitively binding miR-152, contributing to activation of Hh pathway and EMT process in liver fibrosis. We also identify PVT1/ miR-152/PTCH1 as a new signaling network in liver fibrosis (Figure 7).

\section{MATERIALS AND METHODS}

\section{Cells}

Primary HSCs were isolated as described previously [34]. Cells were cultured in Dulbecco's modified Eagle's medium supplemented with $10 \%$ fetal bovine serum, 100 $\mathrm{U} / \mathrm{ml}$ penicillin and $100 \mathrm{U} / \mathrm{ml}$ streptomycin. The purity of cultures was confirmed by immunocytochemical staining for $\alpha$-SMA and the purity reached $>98 \%$. The harvested primary HSCs were studied at day 2 after isolation throughout all the studies. Primary 2-day-old HSCs were transfected with PVT1 siRNA or the scrambled siRNA (siCtrl) using Lipofectamine RNAiMAX (Life Technologies Co, Ltd., Grand Island, NY, USA, cat\# 13778075 ) for $48 \mathrm{~h}$ in all experiments.

\section{$\mathrm{CCl}_{4}$ liver injury model}

Eight-week-old male C57BL/6J mice $(n=6)$ received intraperitoneal injection of $7 \mu \mathrm{L} / \mathrm{g}$ of $10 \% \mathrm{CCl}_{4}$ (Sigma-Aldrich, St. Louis, MO, USA, cat\# 289116) in olive oil two times weekly for six weeks. Meanwhile, mice $(n=6)$ treated with olive oil treatment were considered as the control mice. As well as oil treatment and $\mathrm{CCl}_{4}$ treatment, mice additionally received $\mathrm{CCl}_{4}$ in combination with adenoviral vectors expressing the scrambled shRNA (Ad-shCtrl) $(n=6)$ and $\mathrm{CCl}_{4}$ in combination with AdshPVT1 $(n=6)$ or Ad-shPVT1-2 $(n=6)$. Ad-shPVT1 or Ad-shPVT1-2 $\left(1 \times 10^{9} \mathrm{pfu} / 100 \mu \mathrm{L}\right)$ was injected every two weeks by way of the tail vein for 6 weeks. AdshPVT1, Ad-shPVT1-2 and Ad-shCtrl were purchased from GenePharma biotechnology (Shanghai, China). The animals were provided by the Experimental Animal Center of Wenzhou Medical University. The animal experimental protocol was approved by the University Animal Care and Use Committee. Mice were sacrificed under anesthesia at the end of six weeks and the livers were removed for further analysis. The liver tissues were used for Masson staining by fixation with $10 \%$ formalin. Quantitative analysis for the Masson-positive area was calculated from five fields for each liver slice.

\section{Hepatic hydroxyproline content}

Liver tissues $(50 \mathrm{mg})$ were homogenized in $\mathrm{HCl}$ and hydrolyzed at $120^{\circ} \mathrm{C}$ overnight. After lysate centrifugation at $12,000 \mathrm{~g}$ for $10 \mathrm{~min}$ at $4^{\circ} \mathrm{C}$, the supernatant was evaporated to dryness under vacuum. The hepatic hydroxyproline content was assessed using the Hydroxyproline Colorimetric Assay kit (BioVision, San Francisco, CA, cat\# K555-100). Data were normalized to liver weight.

\section{RNAi assay and transfection analysis}

RNA interference experiments were performed following the manufacturer's protocols. siPVT1 (sense, 5'-AAGGAAGCUCUUCUUGAGC-3'; antisense, 5'GCUC AAGAAGAGCUUCCUU-3'), siPVT1-2 (sense, 5'-GGAAUGCUAAGUUCGUAGC UU-3'; antisense, 5'-AAGCUACGAACUUAGCAUUCC-3') and scrambled siRNA (negative control) were designed and synthesized by GenePharma. miR-152 mimics and miR-152 inhibitor were additionally synthesized by GenePharma. Moreover, siCtrl and miR-NC were used as the negative control. HSCs were transfected with siPVT1, siPVT1-2, miR152 mimics or miR-152 inhibitor using Lipofectamine RNAiMAX at a final concentration of $10 \mathrm{nM}$.

\section{Immunofluorescence microscopy}

Immunofluorescent staining was performed as previously described [8]. Briefly, at $48 \mathrm{~h}$ after primary 2-day-old HSCs were transfected with siPVT1, cells were fixed in an acetic acid: ethanol (1:3) solution for $5 \mathrm{~min}$ at $-20^{\circ} \mathrm{C}$ and washed with $\mathrm{PBS}$. Nonspecific binding was blocked with $5 \%$ goat serum/PBS for $1 \mathrm{~h}$ at 
room temperature, and these cells were then incubated with primary antibodies against E-cadherin (Abcam, Cambridge, MA, USA, cat\# ab76055), desmin (Abcam, cat\# ab15200) or vimentin (Abcam, cat\# ab8978) in a humidified chamber. After washing twice in PBS, the cells were incubated with fluorescein-labelled secondary antibody (1:50 dilution; Dianova, Hamburg, Germany) in antibody dilution solution for $1 \mathrm{~h}$ at room temperature in the dark. The nuclei were stained with 4,6-diamidino2-phenylindole (DAPI) in the dark for $30 \mathrm{~min}$ at room temperature. The slides were washed twice with PBS, covered with DABCO (Sigma-Aldrich), and examined by confocal laser scanning microscopy (Olympus, Tokyo, Japan) at 488 and $568 \mathrm{~nm}$.

\section{Transwell migration assay}

Cells were placed in the top chamber of transwell migration chambers ( $8 \mu \mathrm{m}$; Millipore, Billerica, MA, USA). After $48 \mathrm{~h}$, cells which had not migrated to the lower chamber were removed from the upper surface of the transwell membrane with a cotton swab. Migrating cells on the lower membrane surface were fixed, stained, photographed and counted using a microscope at $\times 100$. Experiments were assayed in triplicate, and $\geq 5$ fields were counted in each experiment.

\section{qRT-PCR}

Total RNA was extracted from cells and liver tissues using the miRNeasy Mini Kit (Qiagen, Valencia, CA, USA, cat\# 217004). 50 ng of total RNA was reversetranscribed to cDNA using the ReverTra Ace qPCR RT Kit (Toyobo, Osaka, Japan, cat\# FSQ-101) in accordance with the manufacturer's instructions. Gene expression was measured by real-time PCR using cDNA, SYBR Green real-time PCR Master Mix (Toyobo, Osaka, Japan, cat\# QPK-101). The PVT1 primers for PCR were listed in Table S1. The primers of Col1A1, $\alpha$-SMA, E-cadherin, vimentin, desmin, PTCH1, SMO, GLI2, U6 and GAPDH were designed as described previously [8]. To detect miR-152 and pri-miR-152 expressions, the RT reactions were performed using the TaqMan MicroRNA Assays (Applied Biosystems, Foster City, CA) according to the manufacturer's instructions. The GAPDH level was used to normalize the relative abundance of PVT1 and mRNAs. U6 snRNA was used to normalize the relative abundance of miRNAs. The expression levels $\left(2^{-\Delta \Delta C t}\right)$ of PVT1, mRNAs and miRNAs were calculated as described previously [35].

\section{Western blot analysis}

Equal amounts of protein were subjected to sodium dodecy1 sulphate-polyacrylamide gel electrophoresis and then transferred onto membranes. The membrane was incubated with primary antibodies overnight at $4^{\circ} \mathrm{C}$, then secondary IRdye800-conjugated goat anti-mouse IgG or goat anti-rabbit IgG at $37^{\circ} \mathrm{C}$ for $1 \mathrm{~h}$. The antibodies are listed in Table S2. Antibody binding was detected using an Odyssey infrared scanner (Li-Cor Biosciences Inc., Lincoln, NE, USA).

\section{Methylation analysis}

PTCH1 CpG island was searched in UCSC Genome Browser. About $0.5 \mu \mathrm{g}$ genomic DNA was treated with sodium bisulfite and subjected to PCR. The PTCH1 primers for PCR were 5'-CTGGGAATTCAAGCCGGACC-3' and 5'-TCTTTCGCTACCGGGAC CT-3'. The bisulfitesequencing analysis was carried out as described previously [36] .

\section{Proliferation assay}

After transfection with siPVT1 and miR-152 inhibitor, HSCs were labelled with EdU for $12 \mathrm{~h}$. HSC proliferative rate was detected using a Cell-Light ${ }^{\mathrm{TM}} \mathrm{EdU}$ In Vitro Imaging Detection Kit (Guangzhou RiboBio Co., Ltd., cat\# C10310-1) according to the manufacturer's instructions.

\section{Luciferase activity assay}

Oligonucleotides containing target sequences of PVT1 were amplified and cloned into pmirGLO plasmids (Promega, Madison, WI, USA). PVT1 for miR-152 forward, 5'- TGCTGTTACCTGTATGCC-3' and reverse, 5'-GCTTCATTACTTAATAAAGC-3'. To obtain the mutated constructs, the fragments harboring the mutated target region were synthesized by GenePharma. Empty plasmid pmirGLO was regarded as a negative control. Luciferase reporter plasmids plus miR-152 mimics or miRNC were co-transfected into $293 \mathrm{~T}$ using Lipofectamine RNAiMAX. Forty-eight hours after transfection, relative luciferase activity was examined in a luminometer using a Dual-Luciferase Reporter Assay System (Promega).

Pull-down assay with Bio-miR-152 Biotin pull-down was performed as previously described $[26,37]$. Briefly, after $48 \mathrm{~h}$ of HSCs transfected with BiomiR-152-wt, Bio-miR-152-mut, or Bio-miR-NC, the cells were washed with PBS followed by incubation in a lysis buffer for $10 \mathrm{~min}$. To exclude RNA and protein complexes, the beads were blocked in lysis buffer including RNasefree bovine serum albumin and yeast tRNA (Sigma). 
After the lysates were incubated with streptavidin-coated magnetic beads (Life Technologies) at $4^{\circ} \mathrm{C}$ for $4 \mathrm{~h}$, they were washed twice with lysis buffer, three times with the low salt buffer, and once with the high salt buffer. The bound RNAs were isolated using TRIzol reagent (Life Technologies). PVT1 expression was determined by qRTPCR.

\section{Statistical analysis}

Data from at least three independent experiments were expressed as the mean \pm SD. Differences between multiple groups were evaluated using one-way analysis of variance. Differences between two groups were compared using a Student's $t$-test. $P<0.05$ was considered significant. All statistical analyses were performed with SPSS software (version 13; SPSS, Chicago, IL).

\section{ACKNOWLEDGMENTS}

The project was supported by the National Natural Science Foundation of China (No. 81500458/H0317), Zhejiang Provincial Natural Science Foundation of China (No. LY16H030012), Guangzhou Science and Technology Project (No.201510010097), and Wenzhou Municipal Science and technology Bureau (No. Y20150091).

\section{CONFLICTS OF INTEREST}

The authors have no conflicts of interest to disclose.

\section{REFERENCES}

1. Zhao Q, Qin CY, Zhao ZH, Fan YC and Wang K. Epigenetic modifications in hepatic stellate cells contribute to liver fibrosis. Tohoku J Exp Med. 2013; 229:35-43.

2. Wang J, Chu ES, Chen HY, Man K, Go MY, Huang XR, Lan HY, Sung JJ and Yu J. microRNA-29b prevents liver fibrosis by attenuating hepatic stellate cell activation and inducing apoptosis through targeting PI3K/AKT pathway. Oncotarget. 2015; 6:7325-7338. doi: 10.18632/ oncotarget.2621.

3. Popov Y and Schuppan D. Targeting liver fibrosis: strategies for development and validation of antifibrotic therapies. Hepatology. 2009; 50:1294-1306.

4. Friedman SL. Hepatic stellate cells: protean, multifunctional, and enigmatic cells of the liver. Physiol Rev. 2008; 88:125-172.

5. Chen Y, Zheng S, Qi D, Guo J, Zhang S and Weng Z. Inhibition of Notch signaling by a gamma-secretase inhibitor attenuates hepatic fibrosis in rats. PLoS One. 2012; 7:e46512.

6. Choi SS, Syn WK, Karaca GF, Omenetti A, Moylan CA, Witek RP, Agboola KM, Jung Y, Michelotti GA and Diehl
AM. Leptin promotes the myofibroblastic phenotype in hepatic stellate cells by activating the hedgehog pathway. J Biol Chem. 2010; 285:36551-36560.

7. Choi SS, Omenetti A, Witek RP, Moylan CA, Syn WK, Jung Y, Yang L, Sudan DL, Sicklick JK, Michelotti GA, Rojkind $\mathrm{M}$ and Diehl AM. Hedgehog pathway activation and epithelial-to-mesenchymal transitions during myofibroblastic transformation of rat hepatic cells in culture and cirrhosis. Am J Physiol Gastrointest Liver Physiol. 2009; 297:G1093-1106.

8. Yu F, Lu Z, Chen B, Wu X, Dong $\mathrm{P}$ and Zheng J. Salvianolic acid B-induced microRNA-152 inhibits liver fibrosis by attenuating DNMT1-mediated Patched1 methylation. J Cell Mol Med. 2015; 19:2617-2632.

9. Yuan JH, Yang F, Wang F, Ma JZ, Guo YJ, Tao QF, Liu F, Pan W, Wang TT, Zhou CC, Wang SB, Wang YZ, Yang Y, Yang N, Zhou WP, Yang GS, et al. A long noncoding RNA activated by TGF-beta promotes the invasion-metastasis cascade in hepatocellular carcinoma. Cancer Cell. 2014; 25:666-681.

10. Fatica A and Bozzoni I. Long non-coding RNAs: new players in cell differentiation and development. Nat Rev Genet. 2014; 15:7-21.

11. He Y, Wu YT, Huang C, Meng XM, Ma TT, Wu BM, Xu FY, Zhang L, Lv XW and Li J. Inhibitory effects of long noncoding RNA MEG3 on hepatic stellate cells activation and liver fibrogenesis. Biochim Biophys Acta. 2014; 1842:2204-2215.

12. Yu F, Zheng J, Mao Y, Dong P, Li G, Lu Z, Guo C, Liu $\mathrm{Z}$ and Fan X. Long non-coding RNA APTR promotes the activation of hepatic stellate cells and the progression of liver fibrosis. Biochem Biophys Res Commun. 2015; 463:679-685.

13. Wang F, Yuan JH, Wang SB, Yang F, Yuan SX, Ye C, Yang N, Zhou WP, Li WL, Li W and Sun SH. Oncofetal long noncoding RNA PVT1 promotes proliferation and stem cell-like property of hepatocellular carcinoma cells by stabilizing NOP2. Hepatology. 2014; 60:1278-1290.

14. Liu E, Liu Z and Zhou Y. Carboplatin-docetaxel-induced activity against ovarian cancer is dependent on up-regulated lncRNA PVT1. Int J Clin Exp Pathol. 2015; 8:3803-3810.

15. Yang YR, Zang SZ, Zhong CL, Li YX, Zhao SS and Feng XJ. Increased expression of the lncRNA PVT1 promotes tumorigenesis in non-small cell lung cancer. Int J Clin Exp Pathol. 2014; 7:6929-6935.

16. Kong R, Zhang EB, Yin DD, You LH, Xu TP, Chen WM, Xia R, Wan L, Sun M, Wang ZX, De W and Zhang ZH. Long noncoding RNA PVT1 indicates a poor prognosis of gastric cancer and promotes cell proliferation through epigenetically regulating p15 and p16. Mol Cancer. 2015; 14:82.

17. Ding C, Yang Z, Lv Z, Du C, Xiao H, Peng C, Cheng S, Xie H, Zhou L, Wu J and Zheng S. Long non-coding RNA PVT1 is associated with tumor progression and predicts 
recurrence in hepatocellular carcinoma patients. Oncol Lett. 2015; 9:955-963.

18. Alvarez ML, Khosroheidari M, Eddy E and Kiefer J. Role of microRNA 1207-5P and its host gene, the long noncoding RNA Pvt1, as mediators of extracellular matrix accumulation in the kidney: implications for diabetic nephropathy. PLoS One. 2013; 8:e77468.

19. Sekiya Y, Ogawa T, Yoshizato K, Ikeda K and Kawada N. Suppression of hepatic stellate cell activation by microRNA-29b. Biochem Biophys Res Commun. 2011; 412:74-79.

20. Ribon V, Johnson JH, Camp HS and Saltiel AR. Thiazolidinediones and insulin resistance: peroxisome proliferatoractivated receptor gamma activation stimulates expression of the CAP gene. Proc Natl Acad Sci U S A. 1998; 95:14751-14756.

21. Yang JJ, Tao H, Huang C, Shi KH, Ma TT, Bian EB, Zhang L, Liu LP, Hu W, Lv XW and Li J. DNA methylation and $\mathrm{MeCP} 2$ regulation of $\mathrm{PTCH} 1$ expression during rats hepatic fibrosis. Cell Signal. 2013; 25:1202-1211.

22. Shi X, Sun M, Liu H, Yao Y and Song Y. Long non-coding RNAs: a new frontier in the study of human diseases. Cancer Lett. 2013; 339:159-166.

23. Sarver AL and Subramanian S. Competing endogenous RNA database. Bioinformation. 2012; 8:731-733.

24. Xing CY, Hu XQ, Xie FY, Yu ZJ, Li HY, Bin Z, Wu JB, Tang LY and Gao SM. Long non-coding RNA HOTAIR modulates c-KIT expression through sponging miR-193a in acute myeloid leukemia. FEBS Lett. 2015; 589:1981-1987.

25. Liang WC, Fu WM, Wong CW, Wang Y, Wang WM, Hu GX, Zhang L, Xiao LJ, Wan DC, Zhang JF and Waye MM. The lncRNA H19 promotes epithelial to mesenchymal transition by functioning as miRNA sponges in colorectal cancer. Oncotarget. 2015; 6:22513-22525. doi: 10.18632/ oncotarget.4154.

26. Yu F, Zheng J, Mao Y, Dong P, Lu Z, Li G, Guo C, Liu Z and Fan X. Long Non-coding RNA Growth Arrest-specific Transcript 5 (GAS5) Inhibits Liver Fibrogenesis through a Mechanism of Competing Endogenous RNA. J Biol Chem. 2015; 290:28286-28298.

27. Wang F, Ying HQ, He BS, Pan YQ, Deng QW, Sun HL, Chen J, Liu X and Wang SK. Upregulated lncRNA-UCA1 contributes to progression of hepatocellular carcinoma through inhibition of miR-216b and activation of FGFR1/ ERK signaling pathway. Oncotarget. 2015; 6:7899-7917. doi: 10.18632/oncotarget.3219.
28. Riquelme E, Suraokar MB, Rodriguez J, Mino B, Lin HY, Rice DC, Tsao A and Wistuba, II. Frequent coamplification and cooperation between C-MYC and PVT1 oncogenes promote malignant pleural mesothelioma. J Thorac Oncol. 2014; 9:998-1007.

29. Paci P, Colombo T and Farina L. Computational analysis identifies a sponge interaction network between long noncoding RNAs and messenger RNAs in human breast cancer. BMC Syst Biol. 2014; 8:83.

30. Benetatos L, Dasoula A, Hatzimichael E, Georgiou I, Syrrou M and Bourantas KL. Promoter hypermethylation of the MEG3 (DLK1/MEG3) imprinted gene in multiple myeloma. Clin Lymphoma Myeloma. 2008; 8:171-175.

31. El Taghdouini A, Sorensen AL, Reiner AH, Coll M, Verhulst S, Mannaerts I, Oie CI, Smedsrod B, Najimi M, Sokal E, Luttun A, Sancho-Bru P, Collas P and van Grunsven LA. Genome-wide analysis of DNA methylation and gene expression patterns in purified, uncultured human liver cells and activated hepatic stellate cells. Oncotarget. 2015; 6:26729-26745. doi: 10.18632/oncotarget.4925.

32. Goll MG and Bestor TH. Eukaryotic cytosine methyltransferases. Annu Rev Biochem. 2005; 74:481-514.

33. Wu X, Zhao B, Cheng Y, Yang Y, Huang C, Meng $\mathrm{X}$, Wu B, Zhang L, Lv X and Li J. Melittin induces PTCH1 expression by down-regulating MeCP2 in human hepatocellular carcinoma SMMC-7721 cells. Toxicol Appl Pharmacol. 2015; 288:74-83.

34. Chang W, Yang M, Song L, Shen K, Wang H, Gao X, Li M, Niu W and Qin X. Isolation and culture of hepatic stellate cells from mouse liver. Acta Biochim Biophys Sin (Shanghai). 2014; 46:291-298.

35. Schmittgen TD and Livak KJ. Analyzing real-time PCR data by the comparative C(T) method. Nat Protoc. 2008; 3:1101-1108.

36. Ji W, Yang L, Yu L, Yuan J, Hu D, Zhang W, Yang J, Pang Y, Li W, Lu J, Fu J, Chen J, Lin Z, Chen W and Zhuang Z. Epigenetic silencing of O6-methylguanine DNA methyltransferase gene in NiS-transformed cells. Carcinogenesis. 2008; 29:1267-1275.

37. Wang K, Liu F, Zhou LY, Long B, Yuan SM, Wang Y, Liu CY, Sun T, Zhang XJ and Li PF. The long noncoding RNA CHRF regulates cardiac hypertrophy by targeting miR-489. Circ Res. 2014; 114:1377-1388. 